\title{
Myasthenic Syndromes - Impact of Rehabilitation and Sports
}

\section{두)(i) (우)}

Author

Thomas Henze

\author{
Affiliation \\ Praxisgemeinschaft für Neurologie, Psychiatrie, Psychother- \\ apie, Regensburg \\ Key words \\ myasthenia gravis, myasthenic syndromes, rehabilitation, \\ sports \\ Bibliography \\ DOI https://doi.org/10.1055/s-0043-120716 \\ Neurology International Open 2018; 2: E1-E5 \\ (c) Georg Thieme Verlag KG Stuttgart · New York \\ ISSN 2511-1795 \\ Correspondence \\ Prof. Dr. Thomas Henze \\ Praxisgemeinschaft für Neurologie \\ Psychiatrie, Psychotherapie, \\ Günzstr. 1 \\ 93059 Regensburg \\ Germany \\ thomas.henze@outlook.com
}

\section{SUMMARY}

Patients suffering from disorders of neuromuscular transmission are only rarely admitted to rehabilitation facilities. However, patients suffering from severe muscular weakness of arms or legs, dysphagia, dysarthria, or respiratory insufficiency, especially after myasthenic crisis, will benefit from rehabilitation. Its goal is maximal reduction of existing disabilities that will enable participation in social life. Besides extensive knowledge of the clinical course and potential complications of myasthenic syndromes, a comprehensive rehabilitation concept is an important prerequisite for successful rehabilitation that includes individual and multimodal physical training as well as coping with the disease, public health medical aspects, education, and assistive devices.

\section{Introduction}

Even though the number of studies evaluating the efficacy of physical exercise and rehabilitation in patients with disorders of neuromuscular transmission is still low, it has become clear that especially in patients well-controlled with medication or in remission physical exertion and increased muscle activity no longer result in prolonged weakness or even persistent damage to skeletal muscles-contrary to the concerns of many of those affected [1].

In general, only few patients living with myasthenia gravis (MG) or another disorder of neuromuscular transmission apply for out-patient or in-patient rehabilitation. This may be due, on the one hand, to fear of irreparable muscle damage with unstable MG, a still widespread misconception, and, on the other hand, to the fact that there is often no need for specific rehabilitative measures in clinically stable patients. According to our experience, many physicians are still unaware that out-patient or in-patient rehabilitation is available for these patients; in addition, there is ongoing uncertainty about the approval practice of health insurances and pension schemes.
Patients experiencing severe clinical courses with significant muscular weakness of arms or legs and/or respiratory muscles as well as problems with swallowing, chewing and speech are frequently unable to lead a normal life, even under optimal pharmacotherapy. According to ICF criteria (ICF = International Classification of Functioning, Disability and Health), these patients suffer from impairments or disabilities in several aspects of life ( $\triangleright$ Tab. 1), affecting their social integration (partner, family, friends, hobbies, etc.), their professional activities and their mental stability.

Thus, these patients need intensive and personalized multimodal rehabilitation, tailored to their individual needs. It is also important that they are regularly followed up at close intervals by the rehabilitation team, monitoring adequate response to treatment using suitable scales. In addition, these patients need to be comprehensively informed and educated about their disease. Rehabilitation, typically of several weeks' duration, also offers the opportunity to optimize symptomatic and immunosuppressive pharmacotherapy.

Given the changeable course of disorders of neuromuscular transmission, the peculiarities of their pharmacotherapy and the 
- Table 1 Impairments and disabilities in patients with myasthenic syndromes, according to ICF.

Mobility

e. g., reduced walking distance and gait stability, including climbing stairs

Activities of daily living

e. g., dressing and undressing, body care and hygiene, household activities

Communication especially fluctuating or permanent dysarthria

Food intake

Respiration e. g., lack of food or fluids, embarrassment by frequent swallowing problems resulting in social withdrawal, risk of aspiration muscular respiratory insufficiency

potential complications, neurological rehabilitation centers treating patients with myasthenic syndromes should be sufficiently experienced in the treatment of these disorders and have the knowledge and facilities required for disease-specific rehabilitation. Preferably, the treatment concept should be truly comprehensive, covering all these aspects. It should also include rarer diseases, such as the Lambert-Eaton syndrome and congenital myasthenic syndromes [2].

\section{Rehabilitation of Patients with Myasthenic Syndromes}

\section{Definition}

According to the definition of D.T. Wade ([3], "rehabilitation is a problem-solving educational process aimed at reducing disability and handicap (participation) experienced by someone as a result of disease or injury".

\section{International classification of functioning, disability \& health (ICF)}

Rehabilitation measures should be based on the ICF, i.e. the disabilities of the patients and the resulting limitations to their participation. The basic consideration is to not treat and improve a symptom (e. g. dysarthria), but rather the disability resulting from this symptom, i. e. in this example the limited communication [4].

To quantify the extent of disabilities, 2 instruments have recently been proposed: the Myasthenia Gravis Activities of Daily Living (MG-ADL) profile [5] and the MG Disability Assessment (MG-DIS [6]). The MG-ADL quantifies 8 activities or the corresponding impairments on a scale from 0 to 3: Speaking, chewing, swallowing, breathing, teeth brushing, getting up from a chair, double vision, and ptosis. This MG-ADL profile correlates well with the Quantitative MG Score (QMG [7]) and the questionnaire designed to measure quality of life in MG (MG-QOL15 [8]). Besides asking questions about generalized, bulbar and ocular symptoms, the MG-DIS covers additional disease-related factors, including fear, sadness, anger, pain, sleeping disturbances and listlessness, and collects more detailed information about impairments resulting from specific myasthenic symptoms [6]. Apart of these impairments per se, their fluctuating severity due to muscle fatigability plays an important role; here, it is distinguished between general fatigability and the worsening of specific impairments [9].

\section{Goals}

The principal aim of rehabilitation is to enable patients to independently and unrestrictedly perform as many as possible of their usual home and professional activities again. Disabilities (measured using MG-ADL or MG-DIS) should be improved or ideally eliminated and, as a result, the quality of life be maintained or enhanced. In addition, potential complications, such as further muscle atrophy due to absent or very limited muscle activity, dysphagia-related aspiration pneumonia and social withdrawal due to problems with speaking and/or swallowing, should be prevented. Another goal of rehabilitation is to support patients by teaching them better coping strategies. The rehabilitation team and the patient should jointly establish the individual rehabilitation goals.

Indications for rehabilitation are summarized in > Table 2 . Rehabilitation is not appropriate or not possible (contraindications) on the one hand in critically ill patients, in particular those with catecholamine-dependent circulatory insufficiency, sepsis, incompletely treated wounds or major comorbidities (general exclusion criteria for neurological early rehabilitation; lack of rehabilitation ability), on the other hand in patients with negative rehabilitation prognosis and those with lack of motivation to participate in rehabilitation (lack of willingness for rehabilitation).

\section{Content of rehabilitation}

Individual and targeted treatment of the existing disabilities (multimodal rehabilitation) is crucial. For this purpose, various functional therapies are available (for an overview, please refer to $>$ Table 3):

- Physiotherapy, sports therapy and medical training therapy, breathing exercises: slow but continuous building-up of muscle strength of arms or legs by strength training and endurance training, improvement of gait stability, including fall prevention, economization of movement patterns and training of the body perception, individual "dosing" of treatment; at the same time, education about targeted exertion-related dosing in daily life and with sports activities.

- Table 2 Indication for rehabilitation in patients with myasthenic syndromes.

- Need for follow-up treatment after a myasthenic or cholinergic crisis, e. g., in case of prolonged weaning, in patients with permanent tracheostomy, with difficult-to-treat dysphagia and/or dysarthria, and with severe paresis of arm and leg muscles

- Imminent respiratory decompensation or chronic respiratory insufficiency, e. g., with nocturnal hypoventilation syndrome

- Need for follow-up treatment after thymectomy [10] and after other surgical procedures where in the their course an increase of myasthenic symptoms has been noted

- Imminent loss of important abilities and/or independence despite out-patient therapies and optimized pharmacotherapy, e. g., gradual reduction of walking distance, increase in bulbar symptoms

- Concomitant diseases with negative impact on symptoms and course of MG, e. g., extensive abdominal or heart-lung-surgery, chronic lung diseases (COPD), psychiatric disorders 
- Table 3 Content of rehabilitation in patients with myasthenic syndromes (overview).

\begin{tabular}{|l|l|}
\hline Primary therapies & \multicolumn{1}{c|}{ Adjuvant therapies/offerings } \\
\hline \begin{tabular}{l|l} 
- Physiotherapy \\
- Sports and training \\
therapy, Training of \\
respiratory muscles
\end{tabular} & - Additional symptomatic therapy (e. g., \\
- - Pain, sleep disturbances) \\
\hline - Speechology therapy & - Relaxation therapies \\
\hline - Swallowing therapy & - Information/education \\
& - Social counselling \\
& - Advice and training on assistive \\
& equipment, if needed \\
& - Cognitive therapy, if needed \\
\hline & - Initiation of home mechanical \\
& ventilation, if needed \\
\hline
\end{tabular}

- Occupational therapy to train activities of daily living: Training of skills to wash and dress themselves, fine motor skills training, etc.

- Speech therapy

- Swallowing therapy with restitutional, compensatory and adaptive techniques after prior clinical assessment and, if required, advanced diagnostic evaluation, such as video-endoscopy (fiberendoscopic evaluation of swallowing, FEES) before and after administration of edrophonium [11]

In addition, there are several other key areas:

- Psychological support, e. g., coping with the disease (coping strategies [12]) and crisis intervention,

- Adjuvant/relaxation techniques, body perception: Feldenkrais method, Qi-Gong, autogenic training, among others,

- Cognitive therapy, where required, if targeted PC-based diagnostic work-up has revealed impairments,

- Treatment of other symptoms, such as pain and sleep disturbances,

- Comprehensive information and education about the disease; information material and education about training programs which can be performed independently,

- Individual assistive devices and adaption, ergonomic advice, if needed

- Potentially initiation and performance of home mechanical ventilation (rarely required), e. g., in close cooperation with a hospital specialized in respiratory medicine,

- Dietary advice, social counselling and family member counselling.

Here, it is important to point out that in neurological early rehabilitation weaning, i. e. the controlled weaning-off of patients with respiratory insufficiency from the ventilator, frequently represents a particular therapeutic challenge and often requires considerable time, as weaning off a patient too quickly can lead to significant deterioration of muscular respiratory exhaustibility.

The course and success of rehabilitation should be monitored using clinical MG scales [7,13], questionnaires on impairments related to activities of daily living $(A D L)[6,9,14]$ and a scale to assess MG-related quality of life [8].
- Table 4 Indications for out-patient rehabilitation.

- Curative treatment, including assistive devices, is not sufficiently successful

- The rehabilitation goal can be achieved by out-patient rehabilitation

- Patient does not require continuous medical monitoring and nursing care

- Support by primary care physician outside of the rehabilitation hours is ensured

- Sufficient mobility and self-care activities

- Sufficient physical and mental stress tolerance

- Travel time to rehabilitation facility is reasonable (up to $30 \mathrm{~min}$.)

- No need to take the patient out of their social environment

- Household support is ensured

Treatment content and quantity is determined during the usually weekly rehabilitation team meetings (ward physician, therapists involved, nurse, and social worker).

\section{Out-patient and in-patient rehabilitation}

During out-patient rehabilitation, patients undergo several functional therapy sessions daily in a rehabilitation facility, tailored to their specific abilities. However, the patient continues to live at home and thus needs to be sufficiently mobile to get to the rehabilitation facility on a daily basis. This type of rehabilitation approach works best in urban areas. During in-patient rehabilitation, patients also undergo several functional therapies daily, but in this case the patient stays as an inpatient at the rehabilitation center over a period of 3 to 4 weeks in general. The indications for out-patient rehabilitation have been summarized in > Table 4 .

All other situations require in-patient rehabilitation, in particular, if the type or extent of damage or the impairments cannot be adequately treated on an out-patient basis, in the presence of severe multimorbidity of various indications, and if nursing care und continuous medical monitoring is necessary (increased need for medical care and need for continuous monitoring and support, including rehabilitation nursing care).

\section{Scientific Data and Studies}

Programs to improve muscular endurance, muscle strength and muscle function have so far been developed primarily for muscular dystrophy and myotonia $[15,16]$. By contrast, very limited scientific data on the effect of muscular training in patients with myasthenic disorders, focusing on differential indication, type and dosing, have been published. However, the few, mostly small studies available indicated that typically an improvement in muscle strength and endurance can be achieved and that this treatment success does not lead to a deterioration of MG.

In 11 patients with mild MG, strength training over a period of 10 weeks (up to 3x/week) improved the maximum force of knee extension, but not of elbow extension (by $23 \%$ [17]). A recently published study evaluating improvement in balance and reduction in risk of falling found in 7 patients with stable myasthenia and unchanged pharmacotherapy that balance training in combination with strength and endurance training plus gradual individualized increase in training intensity resulted in relevant improvements in gait stability and endurance, without any side effects being reported by patients [18]. 
In another recent study, 15 patients with generalized MG underwent either progressive resistance training or aerobic training (20 sessions over a period of 8 weeks). QMG scores did not change over the course of treatment, but some patients reported intermittent bulbar symptoms ( $n=2$ ) or muscular exhaustion $(n=3)$. In the group with progressive resistance training, an increased maximum muscle strength and functional capacity was noted after the end of the study [19].

Patients with respiratory problems may also benefit from regular training. Frequently, myasthenia patients, even if well controlled with medication, show significantly poorer lung function test results compared with healthy subjects (i. e. vital capacity, forced expiratory vital capacity). However, 2 scientific studies showed that regular respiratory-muscle training significantly improved muscle strength and endurance. A controlled study evaluating an 8-week inspiratory muscle training found an improvement in inspiratory muscle strength, an improvement in thoracic wall mobility and an increase in respiratory endurance [20]. An uncontrolled study showed that endurance training of the respiratory muscles resulted in significant improvement in respiratory endurance and an increase in the volume of air ventilated [21].

In addition, a review highlighted that patients should not engage in physical activities and sports when ambient temperatures are at extreme levels and that prolonged intense and unbalanced muscular exercises should be avoided. Exercising should take place after adequate sleep and without stress, ideally at the time of the greatest physical energy as determined on an individual basis [22].

At times, even extreme accomplishments are possible despite myasthenia. For example, a 50-year-old long-distance runner ran weekly distances of approximately $70 \mathrm{~km}$, spread over 5 days, before disease manifestation. Five years after starting this training, he developed MG. Yet, he started again running after he was controlled with medication and even participated at an ultramarathon, i. e. he ran altogether $220 \mathrm{~km}$ on 5 consecutive days, a total of $32 \mathrm{~h}$. He had to increase his daily pyridostigmine dose significantly at these days and repeatedly noticed considerable myasthenic symptoms during the run. He also had to take several additional breaks, but still accomplished to cross the finishing line as the 35th runner out of 65 participants [23].

\section{KEY MESSAGES}

- Patients should undergo rehabilitation if they experience impairments of mobility and/or self-help abilities with activities of daily living, food intake, communication and respiration, if these are not improved by immunosuppressive and/or symptomatic treatment (acetylcholinesterase inhibitors) alone, ideally in centers with proven experience in rehabilitation of myasthenic syndromes.

- Rehabilitation should always be based on a multimodal concept, i. e. involving functional therapies tailored to the impairments of the individual patient, and run long enough to reach the rehabilitation goals established before start of rehabilitation.
- Physical exertion and increased muscle activity do not result in damage to skeletal muscles. On the contrary, the scientific studies published to date showed rather encouraging improvements of strength, muscular endurance, gait stability, and vital capacity after proper training activities.

- For both out-patient and in-patient rehabilitation, clear criteria are available.

\section{Conflict of Interest}

The author declares no conflict of interest.

\section{References}

[1] Westerberg E, Molin C], Lindblad I et al. Physical exercise in myasthenia gravis is safe and improves neuromuscular parameters and physical performance-based measures: A pilot study. Muscle Nerve 2017; 56: 207-214

[2] Henze T, Schumm F, Janzen RWC et al. Rehabilitation bei Patienten mit myasthenen Syndromen - Indikationen, Ziele, Inhalte und erforderliche Strukturen. Akt Neurol 2009; 36: 421-424

[3] Wade DT. Measurement in neurological rehabilitation. Oxford: Oxford University Press; 1992

[4] Schuntermann MF. Einführung in die ICF. 2. Auflage. Landsberg/Lech: Ecomed-Verlag; 2007

[5] Muppidi $S$. The myasthenia gravis-specific activities of daily living profile. Ann N Y Acad Sci 2012; 1274: 114-119

[6] Raggi A, Leonardi M, Schiavolin S et al. Validation of the MG-DIS: A disability assessment for myasthenia gravis. J Neurol 2016; 263: 871-882

[7] Barohn RJ. Reliability testing of the quantitative myasthenia gravis score. Ann N Y Acad Sci 1998; 841: 769-772

[8] Burns TM, Conaway MR, Cutter GR.the Muscle Study Group. Less is more, or almost as much: A 15-item Quality-of-Life instrument for myasthenia gravis. Muscle Nerve 2008; 38: 957-963

[9] Barnett C, Bril V, Kapral M et al. A conceptual framework for evaluating impairments in myasthenia gravis. PLoS ONE 2014; 9: e98089 doi: 10.1371

[10] Ambrogi V, Mineo TC. Benefits of comprehensive rehabilitation therapy in thymectomy for myasthenia gravis: a propensity score matching analysis. Am J Phys Med Rehabil 2017; 96: 77-83

[11] Warnecke T, Teismann I, Zimmermann J et al. Fiberoptic endoscopic evaluation of swallowing with simultaneous tensilon application in diagnosis and therapy of myasthenia gravis. J Neurol 2008; 255: 224-230

[12] Rohr W, Peter H. Verhaltenstherapie bei Myasthenia gravis. Psychother Psychosom Med Psychol 1991; 41: 35-41

[13] Jaretzki A 3rd, Barohn RJ, Ernstoff RM et al. Myasthenia gravis: recommendations for clinical research standards. Task force of the medical scientific advisory board of the myasthenia gravis foundation of america. Neurology 2000; 55: 16-23

[14] Wolfe GI, Herbelin R, Nations SP et al Myasthenia gravis activities of daily living profile. Neurology 1999; 52: 1487-1489

[15] Dawes H, Korpershoek N, Freebody J et al. A pilot randomised controlled trial of a home-based exercise programme aimed at improving endurance and function in adults with neuromuscular disorders. J Neurol Neurosurg Psychiat 2006; 77: 959-962 
[16] Phillips BA, Mastaglia FL. Exercise therapy in patients with myopathy. Curr Opin Neurol 2000; 13: 547-552

[17] Lohi E-L, Lindberg C, Andersen O. Physical training effects in Myasthenia gravis. Arch Phys Med Rehabil 1993; 74: 1178-1180

[18] Wong SH, Nitz JC, Williams K, Brauer SG. Effects of balance strategy training in Myasthenia gravis. A case study series. Muscle Nerve 2014; 49: 654-6604

[19] Rahbek MA, Mikkelsen EE, Overgaard K et al. Exercise in Myasthenia gravis: A feasibility study of aerobic and resistance training. Muscle \& Nerve 2017; 56: 700-709
[20] Fregonezi GA, Resqueti VR, Güell R et al. Effects of a 8-week, interval-based inspiratory muscle training and breathing retraining in patients with generalized Myasthenia gravis. Chest 2005; 128: 1524-1530

[21] Rassler B, Hallebach G, Kalischewski P et al. The effect of respiratory muscle endurance training in patients with myasthenia gravis. Neuromusc Dis 2007; 17: 385-391

[22] Cass S. Myasthenia gravis and sports participation. Curr Sports Med Rep 2013; 12: 18-21

[23] Scheer BV, Valero-Burgos E, Costa R. Myasthenia gravis and endurance exercise. Am J Phys Med Rehabil 2012; 91: 725-727 